\title{
Hypoxia-related Genes as Prognostic Signature to Predict Prognosis and Immune Microenvironment of Colorectal Cancer Patients
}

\section{Yuqin Qiu}

Third Affiliated Hospital

\section{Xuewei Qi}

Third Affiliated Hospital

\section{Zeyu Liu}

Third Affiliated Hospital

Jingnan Xu

Third Affiliated Hospital

\section{Xiaogang Wang}

Department of Emergency Medicine

Jinchang Huang ( $\sim$ zryhhuang@163.com )

1.Third Affiliated Hospital;2.Institute of acupuncture and moxibustion in cancer care

\section{Xin Jiang}

Third Affiliated Hospital

\section{Research}

Keywords: colorectal cancer, hypoxia, immune microenvironment, immunosuppression, prognostic

Posted Date: February 23rd, 2021

DOl: https://doi.org/10.21203/rs.3.rs-219462/v1

License: (9) This work is licensed under a Creative Commons Attribution 4.0 International License. Read Full License 


\section{Abstract}

Background: Hypoxia is widespread in solid tumors and is directly associated with colorectal cancer (CRC) aggressiveness, poor prognosis, and immunotherapy resistance. In this study, we aimed at developing a hypoxia-related marker to improve the prognosis prediction in CRC.

Methods: We used gene expression data of CRC samples from the Cancer Genome Atlas Database and the hypoxia gene set to obtain a hypoxia gene expression matrice of $479 \mathrm{CRC}$ patients. The prognostic model was constructed by screening hypoxia risk genes that were significantly associated with prognosis by univariate and multivariate Cox regression analysis. The predictive performance of the prognostic model was evaluated by Kaplan-Meier survival curve analyses and ROC curve analysis and validated in the GSE17536 dataset of Gene Expression Omnibus database. Finally, we analyzed the immune cell infiltration and expression of immunosuppressive genes in CRC patients at high and low risk of hypoxia.

Results: We constructed a hypoxia risk prognostic model composed of two hypoxia-related genes (SLC2A3 and ENO3), which was proved to have better sensitivity and specificity after a series of validation. Independent prognostic analysis revealed that the risk score can serve as an independent prognostic factor for CRC. The infiltration of natural killer resting cells, activated master cells and T-cell regulatory cells were significantly increased in the hypoxia high-risk group, and Gene Set Enrichment Analysis showed that gene sets involved in tumor proliferation and differentiation, immune tolerance as well as hypoxia were also significantly enriched in this group. Negatively regulated genes in the Cancer Immunity Cycle, as well as immune checkpoints, were upregulated in the high hypoxia risk group, forming an immunosuppressive microenvironment, and mediating the immune escape.

Conclusions: In summary, we constructed and validated a reliable hypoxia risk model that can independently predict the prognosis of CRC patients and reflect the status of the immune microenvironment, which is beneficial for screening CRC prognostic biomarkers and therapeutic targets.

\section{Introduction}

Colorectal cancer (CRC) is one of the malignant tumors that most seriously threaten human life. The morbidity and mortality of CRC are increasing yearly. It has become the third most common cancer in the world and the second leading cause of cancer-related deaths. According to statistics, there will be 1.93 million new CRC cases and 935,000 deaths from CRC worldwide in 2020 [1]. The onset of CRC is insidious, and most patients are already at an advanced stage when diagnosed, which miss the optimal timing of treatment, and their 5-year survival rate is poor [2]. Although some progress has been made in the screening and treatment of $\mathrm{CRC}$ in recent years, the low survival rate, high recurrence rate, and poor prognosis are still not optimistic. Therefore, actively seeking effective biomarkers and therapeutic targets of CRC have positive importance for the early diagnosis, treatment, and improvement of prognosis in patients with CRC. 
Hypoxia is a very common sign of malignant tumor growth, occurring in $90 \%$ of solid tumors, which is a key factor driving tumor invasion and metastasis [3]. In tumors, hypoxia leads to an increase in the activity of hypoxia-inducible factors, thereby promoting tumor angiogenesis, metabolic reprogramming, epithelial-mesenchymal transition, invasion, metastasis, maintenance of tumor stem cells, immune escape, and resistance to radiotherapy and chemotherapy [4]. Numerous studies have shown that hypoxia is a key barrier of the anti-tumor immune response and can mediate immune escape of tumor cells, and also has a direct correlation with tumor aggressiveness. This feature has also been determined as an index of poor prognosis, independent of clinical staging of the tumor [5-6]. Hence, there is an urgent need to find targeted biomarkers of hypoxia. Some studies have established risk models by analyzing the expression of hypoxia-related genes to predict the prognosis of gastric cancer, glioma, hepatocellular carcinoma, and the correlation with the immune microenvironment [7-9]. However, relevant research is not available in CRC.

This study used bioinformatics analysis of the gene expression data in the Cancer Genome Atlas (TCGA) database and the Gene Expression Omnibus (GEO) database to identify hypoxia-related genes that are significantly associated with prognosis and to construct a hypoxia risk model to predict the prognosis and immune microenvironment in patients with CRC, to better guide the clinical treatment of CRC.

\section{Materials And Methods}

\section{Data collection and organization}

The RNA-sequencing transcriptome data and related clinical information of 479 patients with CRC were obtained from the TCGA database (https://portal.gdc.cancer.gov, until November 28, 2020) as a training set. The GSE17536 dataset obtained from the GEO database (https://www.ncbi.nlm.nih.gov/geo, until November 29,2020) was used as a test set, which included clinical information of 177 patients with CRC. The Series Matrix File(s) of the GSE17536 dataset was downloaded, with the probes converted into standard gene names. The data used in this study were obtained from public databases and strictly abided by the publishing guidelines provided by the TCGA. Thus, ethical approval was not required.

\section{Extraction of hypoxia-related genes}

The hypoxia gene set (HALLMARK_HYPOXIA) was downloaded from the Gene Set Enrichment Analysis website (https://www.gsea-msigdb.org/gsea/index.jsp), which contained 200 genes that were upregulated owing to low oxygen levels. We merged these genes with the RNA-seq transcriptome data to extract the expression levels of hypoxia-related genes of the TCGA dataset.

\section{Protein-protein interaction (PPI) network construction}

To determine the interaction between hypoxia-related genes, the genes were mapped to the Search Tool for the Retrieval of Interacting Genes/Proteins (STRING) (https://string-db.org/) database, followed by utilizing Cytoscape (version 3.6.1) for network visualization [10]. The number of connected nodes of 
hypoxia-related genes was calculated and sorted using $\mathrm{R}$ software to select the top 50 genes as the hub genes.

\section{Construction and verification of hypoxia risk model}

Univariate Cox regression analysis was performed on the hub genes in the PPI. The hypoxia-related genes associated with prognosis were selected using a log-rank test $(P<0.01)$. Further multivariate Cox regression analysis was performed on these genes to construct a hypoxia risk model for prognosis and calculate the risk score in order to evaluate the prognosis of CRC patients.

The formula for calculating the risk score of each patient is as follows:

Risk score $=\sum_{i=1}^{n}($ Expi $* \beta i)$ :

where $\beta$ represents the regression coefficient, and Exp represents the gene expression value.

To evaluate and verify the predictive ability of the prognostic model, patients in the training set and the test set were divided into low- and high-risk groups respectively, according to the median risk score of the training set; this was followed by Kaplan-Meier survival analysis to compare the overall survival rate of the two groups of patients. The log-rank test was used to calculate the $P$-value. Receiver operating characteristic (ROC) curves were prepared to compare the prediction accuracy of the prognostic model. Area under the ROC curve (AUC) $>0.6$ was considered an acceptable model.

In order to evaluate and verify the predictive of this prognostic model, we divided the patients in training set and test set into low-risk group and high-risk group according to the median risk score of training set. Kaplan-Meier survival analysis was performed to compare the overall survival rate of the two groups.

Univariate and multivariate Cox regression analyses with risk score and other clinical variables (e.g., age, sex, tumor grading, and cancer staging) were performed to determine whether the risk score could be used as an independent prognostic factor. To further verify the expression of the model genes, the expression level of the model genes was tested using the Human Protein Atlas (HPA) online database (http://www.proteinatlas.org). In addition, a nomogram was drawn based on the hypoxia-related genes in the model, to quantitatively and better predict the survival period of patients with CRC.

\section{Gene set enrichment analysis (GSEA)}

GSEA is a computational method to determine whether an a priori defined set of genes shows statistically significant and concordant differences between two biological states. The Molecular Signatures

Database (MSigDB) is a collection of annotated gene sets for use with GSEA software. To further explore the activated signaling pathways or gene sets in the groups of patients with high-and low-risks of hypoxia, the GSEA software (version 4.1.0) was used to separately analyze enrichment of the gene expression information in the training set and the test set in the MSigDB Collection (h.all.v7.2.symbols.gmt; c5.go.bp.v7.2.symbols.gmt; c2.cp.kegg.v7.2symbols.gmt). 


\section{Correlation with immune cells}

Hypoxia in tumors inhibits the effect of anti-tumor immune cells and mediates immune escape. To further evaluate the immune microenvironment caused by hypoxia, the immune cell infiltration of patient groups with high- and low- risk of hypoxia was analyzed. CIBERSORT (https://cibersort.stanford.edu/) is an analysis tool for calculating the abundance of member cell types in mixed cell populations using gene expression data. Monte Carlo sampling methods are used to calculate the empirical $P$-value of deconvolution to represent the accuracy of the results. $P<0.05$ indicated that the inferred cell composition was more reliable.

The leukocyte gene signature matrix composed of 547 genes, also called LM22, was used to distinguish 22 types of immune cells, including naive B cells, memory B cells, plasma cells, CD8 T cells, naive CD4 T cells, resting memory CD4 T cells, activated memory CD4 T cells, follicular helper T cells, regulatory $T$ cells (Tregs), gamma delta T cells, resting NK cells, activated NK cells, monocytes, M0 macrophages, M1 macrophages, M2 macrophages, resting dendritic cells, activated dendritic cells, resting mast cells, activated mast cells, eosinophils, and neutrophils. First, the CIBERSORT algorithm and the LM22 signature matrix were used to calculate the infiltration of 22 immune cells in the high- and the low-risk hypoxia groups in the training set and the test set, respectively, followed by visually analyzing the immune cells with significant differences in infiltration.

\section{Correlation with immune genes}

The Cancer-Immunity Cycle is the circulatory system in which the immune system recognizes and kills tumor cells in the body. Each step has corresponding positive and negative regulators to ensure activation of the immune system within the normal range. Immune escape of tumor cells is achieved by overriding certain steps of the Cancer-Immunity Cycle. We analyzed the expression of negative regulators in patients with high- and low- hypoxia risk groups. The gene signature set was downloaded from the Tracking Tumor Immunophenotype website (http://biocc.hrbmu.edu.cn/TIP/index.jsp) with the selection "negative" to obtain negative regulatory factors. Immunosuppressive cytokines and immune checkpoints are upregulated under hypoxic conditions. Thus, we also analyzed the expression of these molecules in different hypoxia risk groups.

\section{Results}

\section{Identification of hypoxia-related genes associated with CRC prognosis}

The clinical information and gene expression data of 479 patients with CRC downloaded from the TCGA and the hypoxia-related gene set (HALLMARK_HYPOXIA) downloaded from the GSEA website were combined to obtain the expression matrix of hypoxia-related genes in patients with CRC, including 200 hypoxia-related genes and their expression levels. To further study the interaction between these hypoxia- 
related genes, the STRING database was used to construct a PPI network, followed by visualizing the results with Cytoscape (Fig. 1A). Through the calculation of the number of node connections, the top 50 genes, namely GAPDH, VEGFA, IL6, EGFR, JUN, HK1, LDHA, ENO1, PGK1, ALDOA, TPI1, GPI, FOS, GCK, PFKL, HK2, PYGM, PFKP, PGM1, PKLR, ENO2, GAPDHS, GYS1, SLC2A1, ALDOB, ALDOC, SERPINE1, ENO3, TKTL1, CAV1, CTGF, FBP1, PGAM2, FOXO3, CXCR4, DCN, HMOX1, PFKFB3, SDC2, BGN, CDKN1A, GBE1, GPC3, HSPA5, PDK1, SLC2A3, UGP2, LOX, SDC4, ATF3, were considered the hub genes of the PPI network (Fig. 1B). To study the prognostic significance of these hub genes, univariate Cox regression analysis was performed to obtain the three hypoxia-related genes associated with prognosis, i.e., solute carrier family 2 member 3 (SLC2A3), enolase (ENO)2, and ENO3 (Fig. 1C).

\section{Construction and verification of the hypoxia risk model}

In addition, multivariate Cox regression analysis was performed on prognosis-related genes to obtain two hypoxia-related genes with which to construct a hypoxia risk model for prognosis, i.e., SLC2A3 and ENO3 (Fig. 1D). The corrplot package in $\mathrm{R}$ was also used to analyze the above two models. Correlation analysis revealed no obvious correlation between the two genes (Fig. 1E,F). Table 1 shows the information of the prognosis-related gene models.

Table 1

Two model gene identified by multivariate Cox regression analysis

\begin{tabular}{|llllll|}
\hline Name & Coef & Hazard ratio & Lower $95 \%$ Cl & Upper $95 \%$ Cl & $P$-value \\
\hline SLC2A3 & 0.3139 & 1.3687 & 1.1120 & 1.6847 & 0.0031 \\
ENO3 & 0.9733 & 2.6467 & 1.6270 & 4.3055 & $8.8 \mathrm{e}-05$ \\
\hline
\end{tabular}

To evaluate the predictive power of the prognostic model, the patients in the training set were divided into high- and low-risk hypoxia groups based on the median risk score in survival analysis. The results showed that the survival rate of patients in the high-risk group was significantly poorer than that of patients in the low-risk group (Fig. 2A). ROC analysis was used to test the prediction accuracy of the model. The results showed that the model predicted the prognosis of patients with CRC well. The AUCs of $1-, 3-$, and 5 -year survival rates were $0.639,0.631$, and 0.701 , respectively (Fig. $2 \mathrm{C}$ ).

To further verify the accuracy of the prognostic model, patients in the test set were also divided into highand low-risk hypoxia groups, followed by survival analysis and ROC analysis. The results showed significant differences between patients in the high- and low-risk hypoxia groups (Fig. 2B). The AUCs of $1-, 3-$, and 5 -year survival rates were $0.608,0.605$, and 0.588 (Fig. 2D), respectively, suggesting that the prognostic model had good sensitivity and specificity.

As shown in Fig. 3 depicting the risk-score curves, survival status distribution, risk histograms, and model gene expression heat maps of patients in the high- and low-risk hypoxia groups, as risk score increased, the number of deaths increased in patients with CRC; the expression levels of model genes also increased 
accordingly. These results suggested that there was a more severely hypoxic tumor microenvironment among patients in the high-risk group. The risk histograms showing the survival status of patients in the high- and low-risk groups indicated that the survival rate of patients in the low-risk group was higher than that of the high-risk group, which was also verified in the test set. These results indicated that the prognostic model constructed in this study could well predict the prognosis of patients with CRC.

Univariate Cox regression analysis in the training set suggested that age, stage, TNM classification and risk score were related to the prognosis of patients with CRC (Fig. 2E). Multivariate Cox regression analysis suggested that risk score could be used as an independent risk factor to predict the prognosis of patients with CRC (Fig. 2F). This result was also verified in the training set (Fig. 2G,H).

\section{Model gene expression and nomogram}

To verify differences in the expression of model genes, the immunohistochemical maps of these two genes were obtained from the HPA database. The results showed that the expression of SLC2A3 and ENO3 in CRC was increased, compared with normal colorectal tissue (Fig. 4A,B). A nomogram was constructed based on these two genes, to quantitatively predict the prognosis of patients with CRC. By adding the expression levels of these two genes, the total points were calculated to predict the 1-, 2-, and 3-year survival of patients (Fig. 4C).

\section{Clinical relevance of model gene expression}

Hypoxic microenvironment results in tumors with more aggressive and metastatic features, which are related to poor prognosis for patient. In this study, we investigated the correlation between expression of hypoxia-related genes in the model and the clinical stage of patients with CRC. From the heat map, the expression of SLC2A3 showed significant differences in different stages, with significantly increased in advanced-stage; the expression of ENO3 showed no significant difference in different stages (Fig. 5A,B).Quantitative analysis of model gene expression in the TCGA and GEO datasets confirmed that the expression level of $S L C 2 A 3$ was also increased with the progress of staging (Fig. 5C,D), indicating that the degree of hypoxia was closely related to tumor progression.

\section{GSEA recognizes hypoxia-related signals and gene sets}

To further clarify the activated hypoxia-related signals and gene sets in the high- and low-risk hypoxia groups, GSEA software (version 4.1.0) was used to analyze gene expression information related to the risk of hypoxia in the training set and test set, with normalized enrichment score (NES) $>1$ and nominal $P$ value (NOM $P$-val) $<0.05$ considered to indicate significant gene sets. The results showed that activated hypoxia signals and gene sets were abundant in the high-risk group, mainly related to tumor proliferation, differentiation, immune tolerance, and hypoxia, such as IL2-STAT5 signaling, hypoxia, IL6-JAK-STAT3 signaling, and angiogenesis (Fig. 6A,B). Gene Ontology (GO) enrichment was mainly enriched in the negative regulation of cell-cell adhesion, negative regulation of immune system process, negative regulation of cell activation, and negative regulation of $\mathrm{T}$ cell proliferation (Fig. $6 \mathrm{C}$ ). The Kyoto 
Encyclopedia of Genes and Genomes (KEGG) signaling pathways were mainly enriched in the JAK-STAT signaling pathway, Toll-like receptor (TLR) signaling pathway, and VEGF signaling pathway (Fig. 6D).

\section{Immune cell infiltration in high- and low-risk hypoxia groups}

To clarify the infiltration of immune cells, the CIBERSORT algorithm and LM22 signature matrix were used to calculate differences in the infiltration of 22 immune cell types in high- and low-risk hypoxia groups (Fig. 7A). The infiltration rate of resting natural killer (NK) cells in patients with a high risk of hypoxia was significantly higher than those with a low hypoxia risk. In addition, the infiltration of activated mast cells, Tregs, and neutrophils cells was higher among patients in the high-risk group (Fig. 7B-E). From the above results, immunosuppressive cells were highly infiltrated in high risk hypoxia groups, possibly leading to the formation of a local immunosuppressive tumor microenvironment. Therefore, targeting tumor hypoxia may have important clinical value for improving immunotherapy.

\section{Immune gene expression in high- and low-risk hypoxia groups}

Upregulation of negative regulatory factors in the Cancer-Immunity Cycle causes tumor cells to resist recognition and killing by the immune system and causes immune escape. In this study, we analyzed the expression of these negative regulatory factors in patients with high and low risks of hypoxia. As shown in Fig. 8A and 8B, most negative immune regulatory genes were upregulated in patients with a high risk of hypoxia, indicating that this process of the Cancer-Immunity Cycle was inhibited in the high-risk hypoxia this group. In addition, the expression of immune checkpoints was analyzed in the high- and lowrisk hypoxia groups in this study. The results showed that the expression of immune checkpoints, programmed cell death-1 (PD-1), PD-ligand 1 (PD- $L 1)$, lymphocyte-activation gene 3 ( $L A G 3)$, cytotoxic Tlymphocyte-associated protein 4 (CTLA4), and T-cell immunoglobulin, mucin-domain containing-3 (TIM3), and T-cell immunoreceptor with Ig and ITIM domains (TIGIT) in the high-risk hypoxia group was significantly upregulated (Fig. 8C-H). Through analysis of immune gene expression, the expression of immunosuppressive genes in the high-risk hypoxia group was upregulated to mediate immune escape, leading to a poor prognosis in patients with CRC.

\section{Discussion}

Intratumoral hypoxia is the main driving factor in malignant tumor progression, metastasis, immune escape, and treatment resistance. A recent study has shown that hypoxia inhibits tumor immune response, promotes tumor immune resistance and immune tolerance, and damages the function of antitumor effector cells [11]. Hypoxia reduces the activity of effector cells, such as CD4+, CD8 + T cells, and NK cells and the release of immunostimulatory cytokines, or supports immunosuppressive cells, induces the production and expression of immunosuppressive cytokines and immune checkpoint inhibitors, and interferes with anti-tumor immunotherapeutic effects [5]. Another study also showed that there are obvious hypoxic areas in human CRC [12]. Hypoxia is closely related to poor prognosis in patients with $\mathrm{CRC}$ and an immunosuppressive tumor microenvironment [13-14]. However, owing to the 
lack of effective biomarkers, these factors have not yet been combined with clinical treatment of CRC. Thus, the establishment of a hypoxia prediction model as a biomarker for evaluating the prognosis of patients with CRC has important clinical value. In this study, hypoxia-related genes were identified and were significantly related to prognosis in patients with CRC. These genes were used to construct a reliable risk model to predict the prognosis and status of the immune microenvironment in these patients.

In this study, we analyzed gene expression data of 479 patients with CRC in the TCGA database and finally obtained two hypoxia-related genes (i.e., SLC2A3 and ENO3) that were significantly related to prognosis, according to univariate and multivariate Cox regression analysis. Thus, we used these 2 genes to construct a model for the risk of hypoxia. SLC2A3, also known as glucose transporter 3 (GLUT3), mediates glucose transport from outside cells into the cells and plays a key role in metabolic reprogramming of cancer cells. The high expression of SLC2A3/GLUT3 in tumor cells enhances cellular uptake of glucose, thereby providing energy for the uncontrolled growth of tumor cells. SLC2A3/GLUT3 is stably expressed under hypoxic conditions, and early hypoxia-responsive genes are functionally related to glycolysis that SLC2A3/GLUT3 are involved in [15]. Numerous studies have shown that hypoxia-related SLC2A3/GLUT3 is overexpressed in a variety of malignant tumors, such as stomach, breast, thyroid, and oral squamous cell carcinoma, and is related to the clinicopathological characteristics, malignant potential, and poor prognosis of these cancers [16-19]. Kim et al. [20] showed that high SLC2A3 expression indicates a poor prognosis in patients with CRC. The present study also showed that $S L C 2 A 3$ was positively correlated with the risk score of patients with CRC, suggesting that SLC2A3/GLUT3 may be a new biomarker or potential therapeutic target for CRC. ENOs are the main enzymes in the glycolysis pathway, catalyzing the conversion of 2-phosphoglyceride into phosphoenolpyruvate. ENOs in mammalian cells are composed of three subunits: $\alpha$-enolase (ENO1), $\gamma$-enolase (ENO2), and $\beta$-enolase (ENO3). ENO1 is widely distributed throughout a variety of tissues and is related to cancers, such as lung cancer and bladder cancer [21-22]. ENO2 and ENO3 are present in neuron/neuroendocrine and muscle tissues, respectively. Studies have shown that the levels of ENO2 and ENO3 in CRC tissues are higher than those in normal tissues, and the low expression of ENO2 and ENO3 is related to the positive prognosis of CRC [23]. Moreover, downregulation of the ENO3 gene and protein inhibits the growth of tumor cells [24].

In this study, we further analyzed the accuracy and stability of the model through survival analyses, risk histograms, and risk curves. The results showed that the model accurately distinguished between highrisk and low-risk patients. ROC curve analysis also demonstrated that the model accurately predicted the prognostic risk of patients with CRC. This was also verified in the GEO test set, and the results supported the above conclusion. Univariate and multivariate Cox regression analyses showed that the risk model in this study independently predicted the prognosis of patients with CRC. The relationship between expression of hypoxia-related genes in the model and clinical staging was also analyzed. The results showed that the expression of SLC2A3 was related to the progression of CRC. In addition, a nomogram based on our model was constructed to more intuitively predict the prognosis of patients with CRC. The HPA database was used to verify the expression of two hypoxia-related genes. The results showed that compared with normal colon tissue, the expression of SCL2A3 and ENO3 was upregulated in patients 
with $\mathrm{CRC}$, suggesting that CRC tissues had obvious hypoxia. The high expression of SLC2A3 and ENO3 may be a potential carcinogenic risk. In brief, our hypoxia prediction model was relatively reliable and able to identify the risk and prognosis of hypoxia in patients with CRC, which is conducive to early intervention and treatment of these patients.

Our results showed that signaling pathways related to hypoxia, angiogenesis, and immune tolerance, such as the JAK-STAT, TLR, and VEGF signaling pathways had different degrees of enrichment in patients with a high risk of hypoxia. The Janus kinase (JAK)/signal transducer and activator of transcription (STAT) signaling pathway is a signal transduction pathway stimulated by cytokines and is involved in many important biological processes, such as cell proliferation, differentiation, apoptosis, and immune regulation. Abnormal activation contributes to tumorigenesis, metastasis, and tumor angiogenesis [25]. The TLR signaling pathway plays an important role in innate immune responses and antigen-specific acquired immunity. Abnormal activation of the TLR signaling pathway leads to activation of nuclear factor kappa B (NF-KB) signaling and the overexpression of inflammatory cytokines, which are closely related to the abnormal cell proliferation, occurrence, invasion, and metastasis of CRC and are involved in regulating the immunosuppressive effects of Tregs [26-27]. GO analysis in this study showed that hypoxia-related genes associated with CRC prognosis were involved in biological functions, such as cell activation, cell adhesion, negative regulation of the immune system, and T cell differentiation.

Intratumoral hypoxia and immunity are related to the prognosis of patients with cancer. The hypoxic area of solid tumors is infiltrated by a large number of immunosuppressive cells, such as myeloid derived suppressor cells, tumor-associated macrophages, and Tregs [28]. The infiltration of immune cells in the tumor microenvironment affects prognosis in patients with cancer. Fakih et al. [29] showed that there were a large number of $C D 8+T$ cell infiltrations and macrophages with high $P D-L 1$ expression in the CRC patients with poor prognosis; in addition, immune response-related genes and immune checkpoint genes were also overexpressed in CRC tissues. Ge et al. [30] showed that the degree of infiltration of M0 macrophages, M1 macrophages, and memory CD4 T cells in CRC was significantly higher than that of normal tissues. The degree of infiltration among different immunosuppressive cells is related to the course of the disease. We evaluated the correlation between hypoxia risk and the immune microenvironment in CRC and immune cell infiltration in different groups of patients with high and low hypoxia risk; we showed that NK resting cells in patients with a high risk of hypoxia were significantly increased. NK cells are important cytotoxic cells of the immune system. However, owing to factors such as hypoxia and acidic metabolites, the activation and effector functions of NK cells are limited [31]. Studies have shown that decreased NK cell activity in patients with CRC is related to advanced disease and poor postoperative prognosis [32-33]. In addition, we found that activated mast cells, Tregs and neutrophils cells showed a high infiltration state in the high-risk hypoxia group, which also verified that hypoxia caused an immunosuppressive microenvironment. Most studies have shown that increased infiltration of mast cells is directly related to tumor aggressiveness and decreased patient survival [3435]. Tregs is a type of immunosuppressive T cell. Another study showed a positive correlation between Tregs and hypoxia-inducible factor-1 a [36]. The relative risk of recurrence and death in patients with CRC 
who have a high degree of invasion of tumor-stromal Tregs is 12-fold that of patients with a low degree of invasion [37].

In 2013, Chen and Mellman proposed the concept of the Cancer-Immunity Cycle. The immune system must initiate a series of processes to kill tumor cells, through the release of antigen in tumor cells, tumor antigen presentation, $\mathrm{T}$ cell activation and migration to tumor tissues, $\mathrm{T}$ cell infiltration, tumor recognition in tissues, and other steps to eventually eliminate tumor cells [38]. Every step in the Cancer-Immunity Cycle requires the coordination of many factors, including stimulating factors and inhibitors. Immune escape in tumor cells is achieved by overriding certain steps in the cycle. In this study, when exploring the expression of inhibitors in the Cancer-Immunity Cycle, we found that most immune negative regulatory genes were upregulated in patients at high risk of hypoxia, indicating that hypoxia inhibited the CancerImmunity Cycle and mediated tumor immune escape. Immune checkpoints have now become a potential target for cancer treatment. Immunotherapy represented by $P D-1 / P D-L 1$ inhibitors has changed the treatment methods and prognosis of many cancers. Studies have shown that hypoxia upregulates a variety of immune checkpoints, including PD-1, CTLA4, and TIM-3 [39-40]. Barsoum et al. [41] showed that hypoxia upregulated $P D-L 1$ expression on the surface of human breast cancer cells and prostate cancer cells and in mouse melanoma cells and breast cancer cells. Our results also showed that the expression of immune checkpoints PD1, PD-L1, LAG3, CTLA4, TIM-3 and TIGIT were significantly upregulated in $\mathrm{CRC}$ patients with a high risk of hypoxia.

In general, the hypoxia-risk model constructed in this study, based on two hypoxia-related genes associated with prognosis, predicted the prognosis of patients with CRC well and can help clinicians to make clinical decisions. However, this study still has certain limitations. First, the hypoxia-risk model constructed in this study was obtained based on the public clinical databases TCGA and GEO; therefore, the model should be verified in a larger clinical cohort in the future. Second, as to the specific role of the selected hypoxia-related genes associated with prognosis of CRC, in vitro and animal experiments are needed in the future to reveal the underlying mechanism.

\section{Conclusion}

In this study, we systematically analyzed the relationship between hypoxia and the immune microenvironment and prognosis in CRC. For the first time, we constructed and verified a prognostic model based on two hypoxia-related genes. Survival analysis and independent prognostic analysis confirmed that the prognostic model could serve as an independent predictor of prognosis in patients with CRC. The findings of this study can facilitate further research into the mechanism of tumorigenesis and progression of $\mathrm{CRC}$ and have certain guiding importance with respect to clinical prognostic biomarkers and hypoxia-targeted therapy in patients with CRC.

\section{Abbreviations}

CRC Colorectal cancer 
TCGA The Cancer Genome Atlas

GEO Gene Expression Omnibus

ROC Receiver operating characteristic

GSEA Gene set enrichment analysis

Tregs Regulatory T cells

SLC2A3 Solute carrier family 2 member 3

ENO Enolase

GO Gene Ontology

KEGG Kyoto Encyclopedia of Genes and Genomes

TLR Toll-like receptor

NK Natural killer

PD-1 Programmed cell death-1

PD-L1 PD-ligand 1

LAG3 Lymphocyte-activation gene 3

CTLA4 Cytotoxic T-lymphocyte-associated protein 4

TIM-3 T-cell immunoglobulin, mucin-domain containing-3

TIGIT T-cell immunoreceptor with Ig and ITIM domains

JAK/STAT Janus kinase/signal transducer and activator of transcription

NF-кB Nuclear factor kappa B

\section{Declarations}

\section{ETHICS APPROVAL AND CONSENT TO PARTICIPATE}

The data of the patients in this study were obtained from the public database datasets, and written informed consent was obtained from these patients. 
All authors approved the manuscript and consent publication

\section{AVAILABILITY OF DATA AND MATERIALS}

The authors declare that the data supporting the findings of this study are available in the TCGA database (https://portal.gdc.cancer.gov) and GEO database (https://www.ncbi.nlm.nih.gov/geo).

\section{COMPETING INTERESTS}

The authors declare that the research was conducted in the absence of any commercial or financial relationships that could be construed as a potential conflict of interest.

\section{FUNDING}

This work was supported by Beijing University of Chinese Medicine 2020 Basic Research Business Expenses Project (No.2020-JYB-ZDGG-143-1) and National Natural Science Foundation of China General Program (No.82074545).

\section{AUTHORS' CONTRIBUTIONS}

$\mathrm{JH}$ and $\mathrm{XJ}$ conceived and designed the study; JX and XW downloaded and organized the TCGA and GEO data; $Y Q, X Q$ and $L Z$ performed data analysis; $Y Q$ and $X Q$ wrote the paper; $J H$ and $X J$ critically revised the article for essential intellectual content and administrative support. All authors read and approved the final manuscript.

\section{ACKNOWLEDGMENTS}

The authors would like to thank the TCGA, GEO, STRING and HPA databases for the availability of the data.

\section{References}

1. Latest global cancer data: Cancer burden rises to 19.3 million new cases and 10.0 million cancer deaths in 2020. https://www.iarc.fr/fr/news-events/latest-global-cancer-data-cancer-burden-rises-to19-3-million-new-cases-and-10-0-million-cancer-deaths-in-2020/ (archived on December 15, 2020)

2. Hou P, Shi P, Jiang T, Yin H, Chu S, Shi M, et al. DKC1 enhances angiogenesis by promoting HIF-1a transcription and fa-cilitates metastasis in colorectal cancer. Br J Cancer. 2020;122:668-679.

3. Vaupel P, Mayer A, Hckel M. Tumor Hypoxia and Malignant Progression. Methods in Enzymology. 2004;381:335-354.

4. Schito L, Semenza GL. Hypoxia-Inducible Factors: Master Regulators of Cancer Progression. Trends Cancer. 2016;2:758-770.

5. Multhoff G, Vaupel P. Hypoxia Compromises Anti-Cancer Immune Responses. Adv Exp Med Biol. 2020;1232:131-143. 
6. Vito A, El-Sayes N, Mossman K. Hypoxia-Driven Immune Escape in the Tumor Microenvironment. Cells. 2020;9:992.

7. Liu Y, Wu J, Huang W, Weng S, Wang B, Chen Y, et al. Development and validation of a hypoxiaimmune-based micro-environment gene signature for risk stratification in gastric cancer. J Transl Med. 2020;18:201.

8. Zhang B, Tang B, Gao J, Li J, Kong L, Qin L. A hypoxia-related signature for clinically predicting diagnosis, prognosis and immune microenvironment of hepatocellular carcinoma patients. J Transl Med. 2020;18:342.

9. Lin W, Wu S, Chen X, Ye Y, Weng Y, Pan Y, et al. Characterization of Hypoxia Signature to Evaluate the Tumor Immune Microenvironment and Predict Prognosis in Glioma Groups. Front. Oncol. 2020;10:796.

10. Shannon P, Markiel A, Ozier O, Baliga NS, Wang JT, Ramage D, et al. Cytoscape: A software Environment for integrated models of biomolecular interaction networks. Genome Res. 2003;13:2498-2504.

11. Noman MZ, Hasmim M, Messai Y, Terry S, Kieda C, Janji B, et al. Hypoxia: a key player in antitumor immune response. A Review in the Theme: Cellular Responses to Hypoxia. Am J Physiol Cell Physiol. 2015;309:C569-79.

12. Leith JT, Padfield G, Faulkner L, Michelson S. Hypoxic fractions in xenografted human colon tumors. Cancer Res. 1991;51:5139-43.

13. Qi L, Chen J, Yang Y, Hu W. Hypoxia Correlates with Poor Survival and M2 Macrophage Infiltration in Colorectal Cancer. Front Oncol. 2020;10:566430.

14. Westendorf AM, Skibbe K, Adamczyk A, Buer J, Geffers R, Hansen W, et al. Hypoxia Enhances Immunosuppression by Inhibiting CD4+ Effector T Cell Function and Promoting Treg Activity. Cell Physiol Biochem. 2017;41:1271-1284.

15. Mimura I, Nangaku M, Kanki Y, Tsutsumi S, Inoue T, Kohro T, et al. Dynamic change of chromatin conformation in re-sponse to hypoxia enhances the expression of GLUT3 (SLC2A3) by cooperative interaction of hypoxia-inducible factor 1 and KDM3A. Mol Cell Biol. 2012;32:3018-32.

16. Krzeslak A, Wojcik-Krowiranda K, Forma E, Jozwiak P, Romanowicz H, Bienkiewicz A, et al. Expression of GLUT1 and GLUT3 glucose transporters in endometrial and breast cancers. Pathol Oncol Res. 2012;18:721-8.

17. Ayala FR, Rocha RM, Carvalho KC, Carvalho AL, da Cunha IW, Lourenço SV, et al. GLUT1 and GLUT3 as potential prognostic markers for Oral Squamous Cell Carcinoma. Molecules. 2010;15:2374-87.

18. Schlößer HA, Drebber U, Urbanski A, Haase S, Baltin C, Berlth F, et al. Glucose transporters 1, 3, 6, and 10 are expressed in gastric cancer and glucose transporter 3 is associated with UICC stage and survival. Gastric Cancer. 2017;20:83-91.

19. Jóźwiak P, Krześlak A, Pomorski L, Lipińska A. Expression of hypoxia-related glucose transporters GLUT1 and GLUT3 in benign, malignant and non-neoplastic thyroid lesions. Mol Med Rep. 2012;6:601-6. 
20. Kim E, Jung S, Park WS, Lee JH, Shin R, Heo SC, et al. Upregulation of SLC2A3 gene and prognosis in colorectal carcinoma: analysis of TCGA data. BMC Cancer. 2019;19:302.

21. Ho JA, Chang HC, Shih NY, Wu LC, Chang YF, Chen CC, et al. Diagnostic detection of human lung cancer-associated antigen using a gold nanoparticle-based electrochemical immunosensor. Anal Chem. 2010;82:5944-50.

22. Ji M, Wang Z, Chen J, Gu L, Chen M, Ding Y, et al. Up-regulated ENO1 promotes the bladder cancer cell growth and proliferation via regulating $\beta$-catenin. Biosci Rep. 2019;39:BSR20190503.

23. Pan X, Wu H, Chen G, Li W. Prognostic Value of Enolase Gene Family in Colon Cancer. Med Sci Monit. 2020;26:e922980-1-e922980-18.

24. Kong KW, Abdul Aziz A, Razali N, Aminuddin N, Mat Junit S. Antioxidant-rich leaf extract of Barringtonia racemosa sig-nificantly alters the in vitro expression of genes encoding enzymes that are involved in methylglyoxal degradation III. PeerJ. 2016;4:e2379.

25. Bose S, Banerjee S, Mondal A, Chakraborty U, Pumarol J, Croley CR, et al. Targeting the JAK/STAT Signaling Pathway Using Phytocompounds for Cancer Prevention and Therapy. Cells. 2020;9:1451.

26. Moradi-Marjaneh R, Hassanian SM, Fiuji H, Soleimanpour S, Ferns GA, Avan A, et al. Toll like receptor signaling pathway as a potential therapeutic target in colorectal cancer. J Cell Physiol. 2018;233:5613-5622.

27. O'Leary DP, Bhatt L, Woolley JF, Gough DR, Wang JH, Cotter TG, et al. TLR-4 signalling accelerates colon cancer cell ad-hesion via NF-KB mediated transcriptional up-regulation of Nox-1. PLoS One. 2012;7:e44176.

28. Noman MZ, Messai Y, Carre T, Akalay I, Meron M, Janji B, et al. Microenvironmental hypoxia orchestrating the cell stroma cross talk, tumor progression and antitumor response. Crit Rev Immunol. 2011;31:357-77.

29. Fakih M, Ouyang C, Wang C, Tu TY, Gozo MC, Cho M, et al. Immune overdrive signature in colorectal tumor subset predicts poor clinical outcome. J Clin Invest. 2019;129:4464-4476.

30. Ge P, Wang W, Li L, Zhang G, Gao Z, Tang Z, et al. Profiles of immune cell infiltration and immunerelated genes in the tumor microenvironment of colorectal cancer. Biomed Pharmacother. 2019;118:109228.

31. Terrén I, Orrantia A, Vitallé J, Zenarruzabeitia O, Borrego F. NK Cell Metabolism and Tumor Microenvironment. Front Immunol. 2019;10:2278.

32. Tartter PI, Steinberg B, Barron DM, Martinelli G. The prognostic significance of natural killer cytotoxicity in patients with colorectal cancer. Arch Surg. 1987;122:1264-8.

33. Kondo E, Koda K, Takiguchi N, Oda K, Seike K, Ishizuka M, et al. Preoperative natural killer cell activity as a prognostic factor for distant metastasis following surgery for colon cancer. Dig Surg. 2003;20:445-51.

34. Fisher ER, Paik SM, Rockette H, Jones J, Caplan R, Fisher B. Prognostic significance of eosinophils and mast cells in rectal cancer: findings from the National Surgical Adjuvant Breast and Bowel Project (protocol R-01). Hum Pathol. 1989;20:159-63. 
35. Acikalin MF, Oner U, Topçu I, Yaşar B, Kiper H, Colak E. Tumour angiogenesis and mast cell density in the prognostic assessment of colorectal carcinomas. Dig Liver Dis. 2005;37:162-9.

36. Deng B, Zhu JM, Wang Y, Liu TT, Ding YB, Xiao WM, et al. Intratumor hypoxia promotes immune tolerance by inducing regulatory T cells via TGF- $\beta 1$ in gastric cancer. PLoS One. 2013;8:e63777.

37. Waniczek D, Lorenc Z, Śnietura M, Wesecki M, Kopec A, Muc-Wierzgoń M. Tumor-Associated Macrophages and Regu-latory T Cells Infiltration and the Clinical Outcome in Colorectal Cancer. Arch Immunol Ther Exp (Warsz). 2017;65:445-454.

38. Chen DS, Mellman I. Oncology meets immunology: the cancer-immunity cycle. Immunity. 2013;39:110.

39. Ohta A. Oxygen-dependent regulation of immune checkpoint mechanisms. Int Immunol. 2018;30:335-343.

40. Koh HS, Chang CY, Jeon SB, Yoon HJ, Ahn YH, Kim HS, et al. The HIF-1/glial TIM-3 axis controls inflammation-associated brain damage under hypoxia. Nat Commun. 2015;6:6340.

41. Barsoum IB, Smallwood CA, Siemens DR, Graham CH. A mechanism of hypoxia-mediated escape from adaptive immunity in cancer cells. Cancer Res. 2014;74:665-74.

\section{Figures}


A

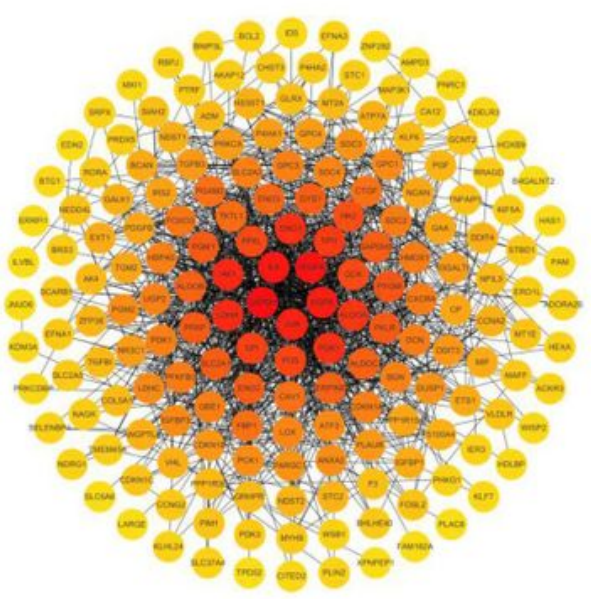

C

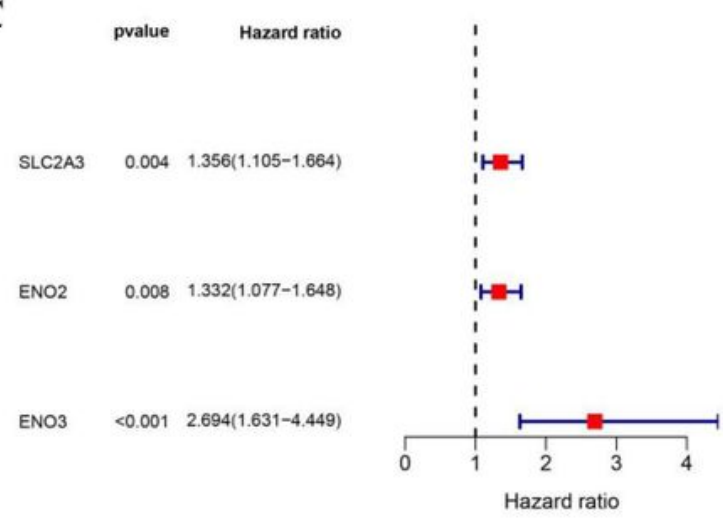

E

\section{TCGA}

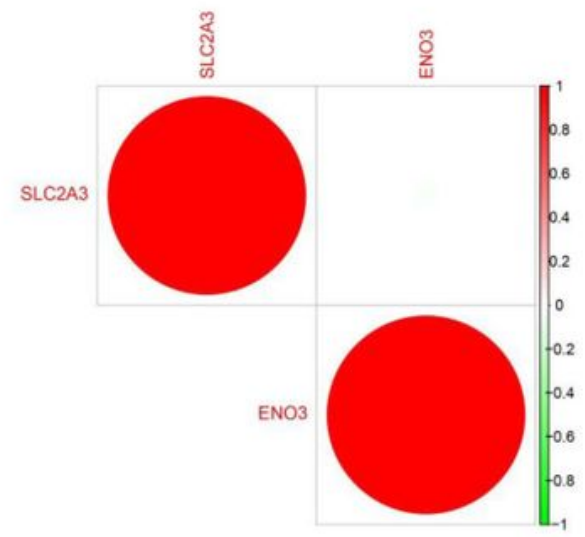

B

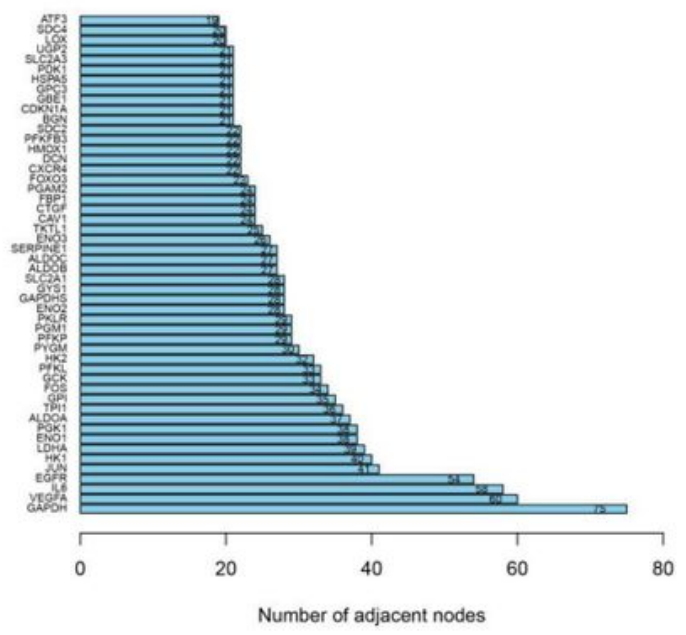

D

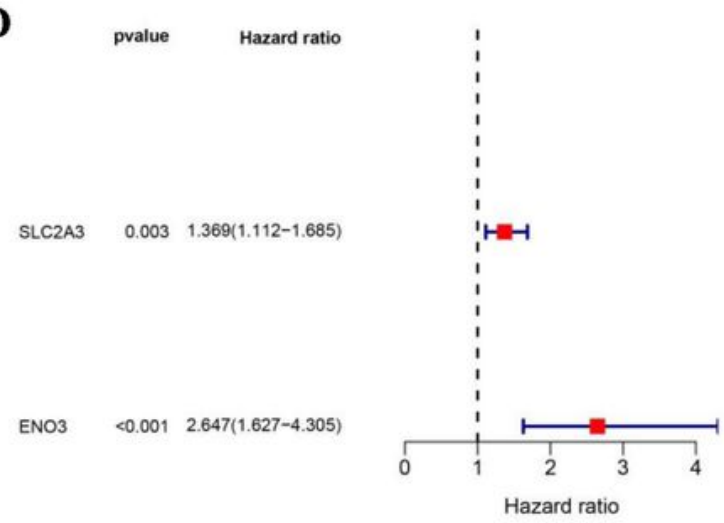

$\mathbf{F}$ GEO

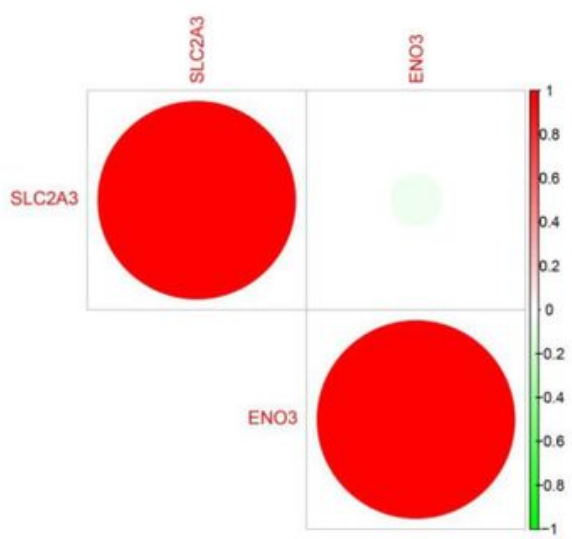

\section{Figure 1}

Selection of prognosis-related hypoxia gene. (A) Protein-protein interaction (PPI) network among 200 hypoxia-associated gene; (B) Interaction degree of Top 50 hypoxia-associated gene; The (C) univariate and (D) multivariate Cox regression analysis of constructing the hypoxia risk prognostic model; $(E, F)$ Spearman correlation of two model gene in TCGA and GEO database. 
A

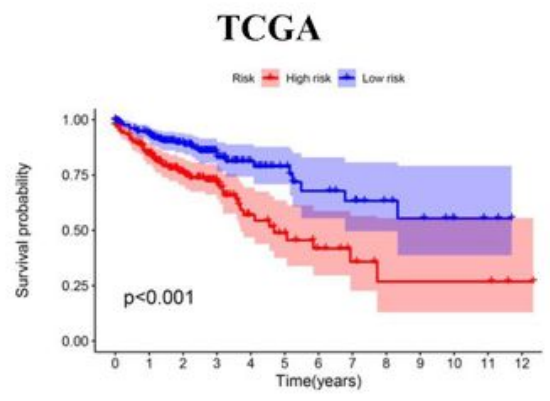

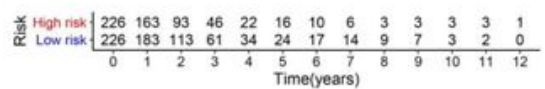

C

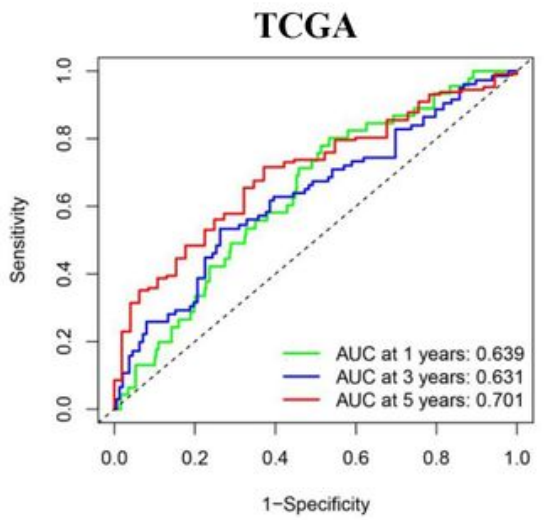

E

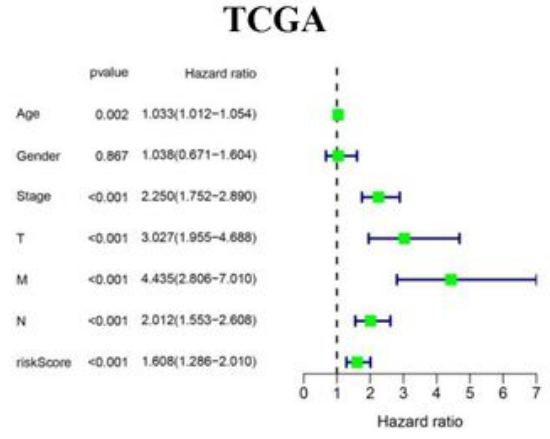

G

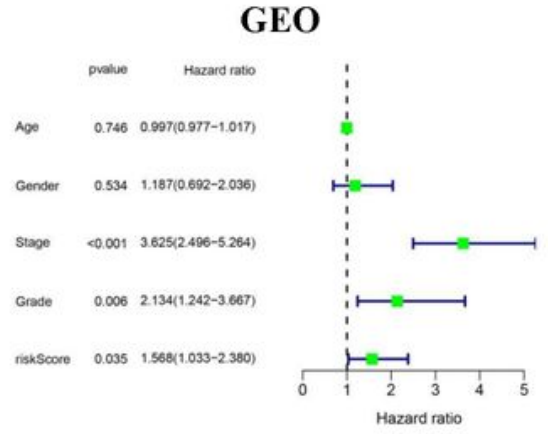

B

GEO

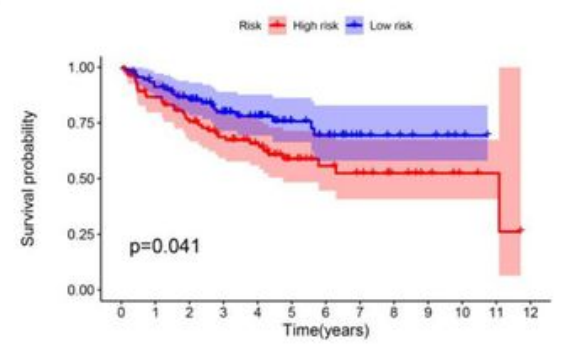

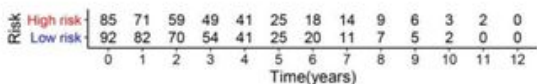

D

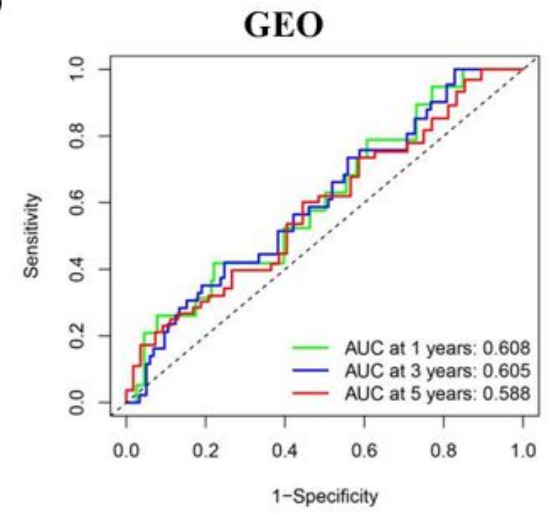

F

TCGA

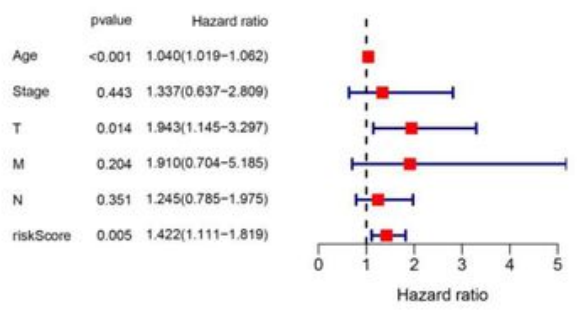

H

\section{GEO}

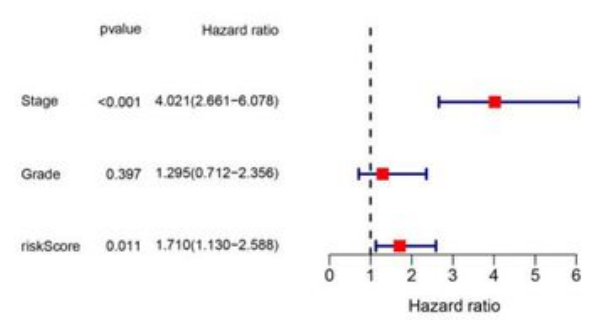

Figure 2

Risk score analysis of hypoxia risk prognostic model. (A, B) Kaplan-Meier curves and (C, D) ROC analysis of the prognostic model; The $(E, G)$ univariate and $(F, H)$ multivariate Cox regression analysis of evaluating the independent prognostic value of the risk score of the prediction model. 
A

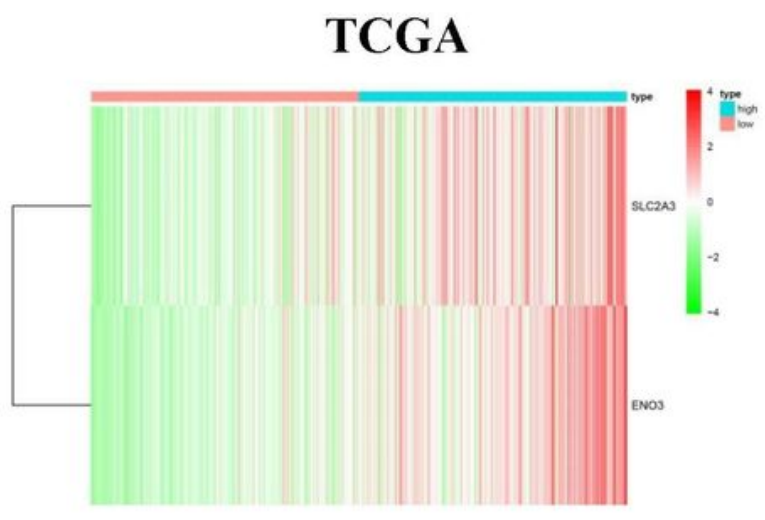

C

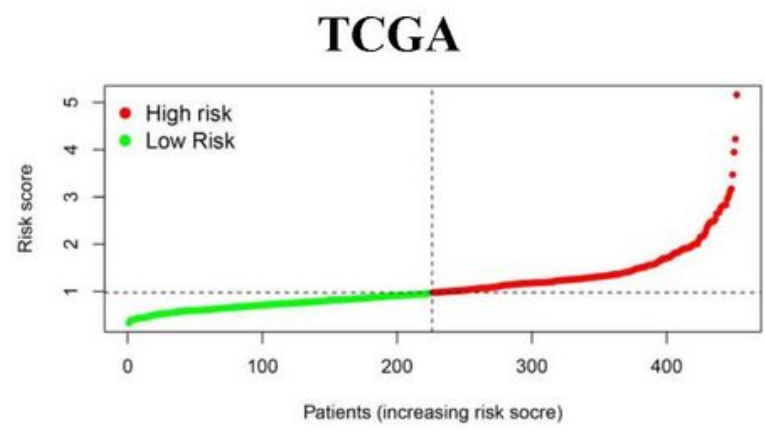

E

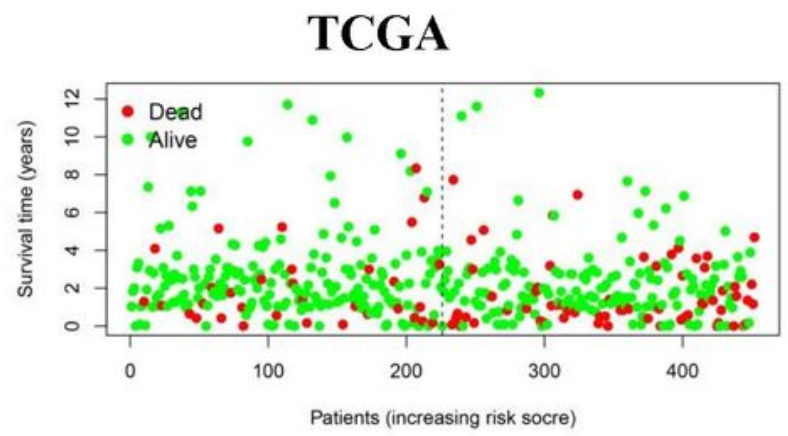

G

TCGA

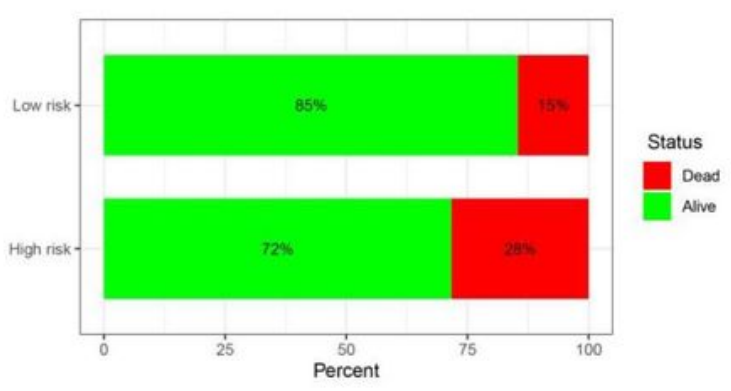

B

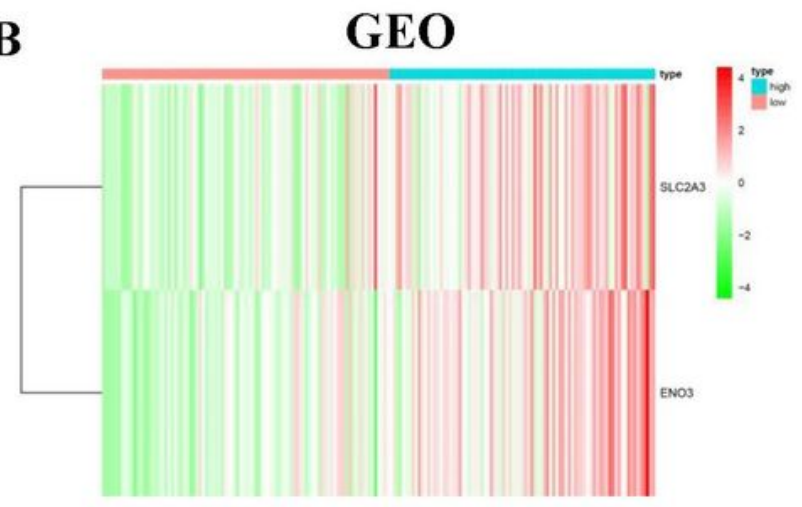

D

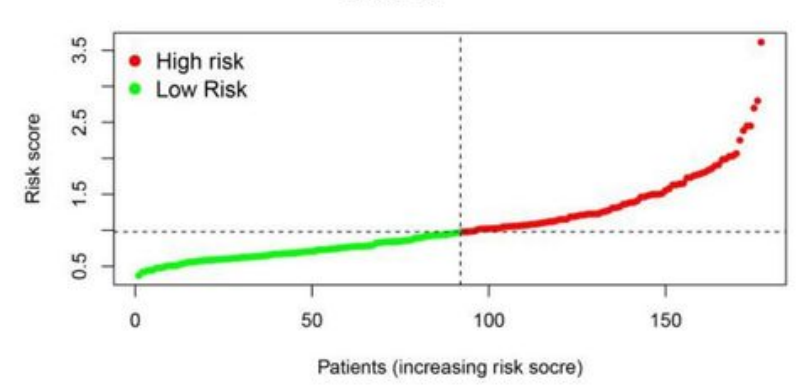

F

GEO

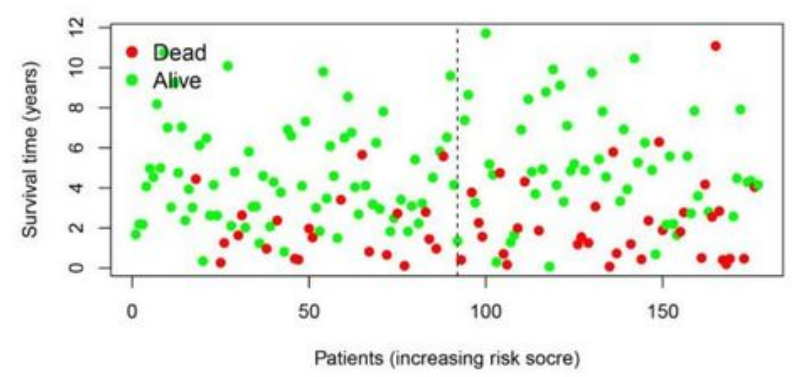

H

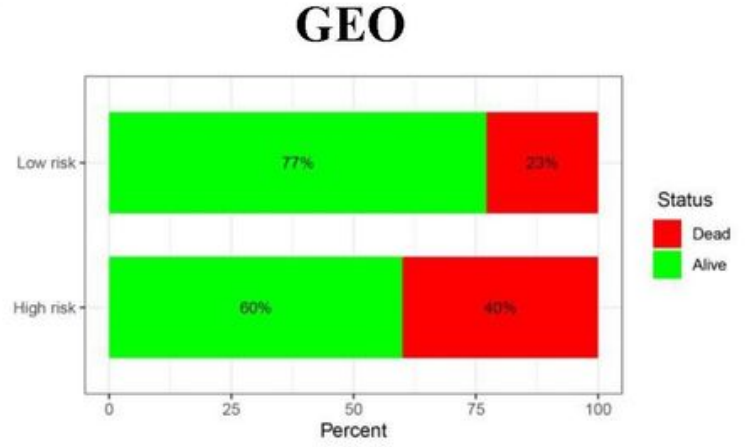

\section{Figure 3}

Prognostic value of the hypoxia risk prognostic model. (A, B) heat map, $(C, D)$ risk score curves, $(E, F)$ survival status and $(G, H)$ mortality rate of $C R C$ patients in TCGA and GEO database. 
A
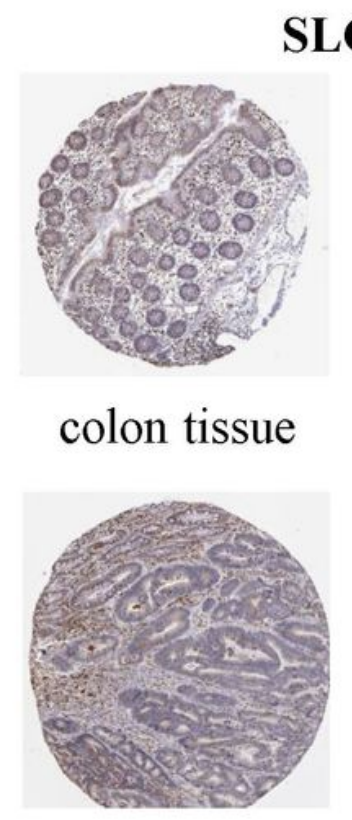

colon cancer
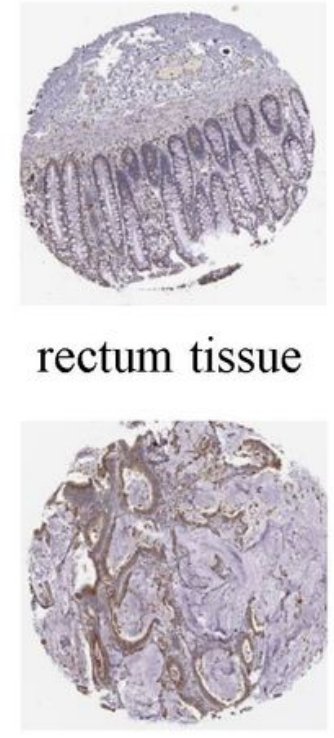

rectal cancer
B

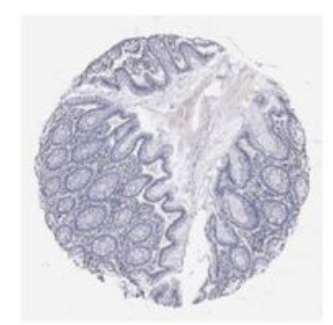

colon tissue

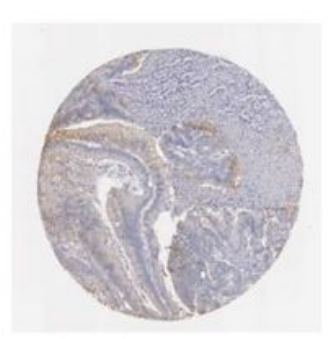

colon cancer
ENO3

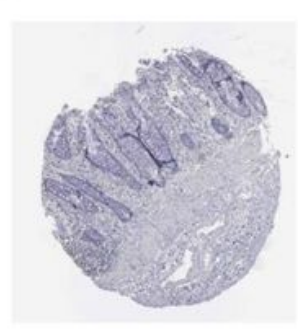

rectum tissue

C

Points

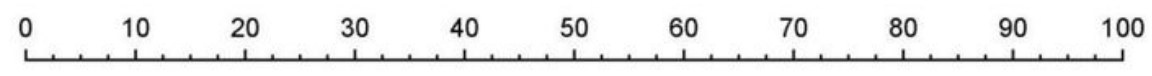

SLC2A3

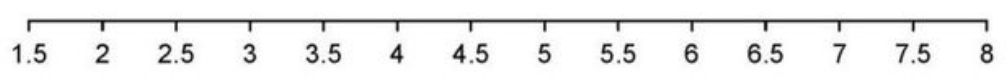

ENO3

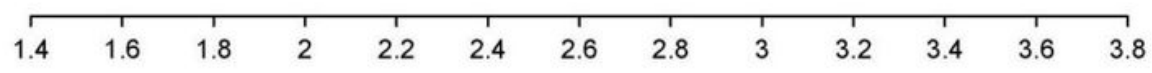

Total Points

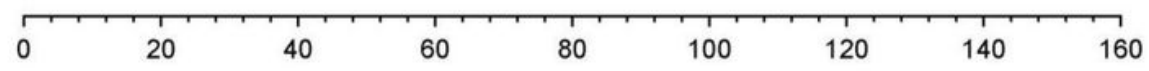

1-year survival

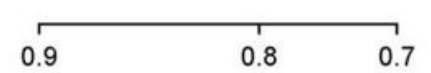

2-year survival

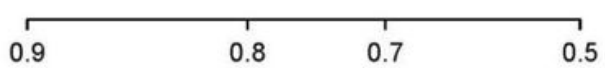

3-year survival

\begin{tabular}{lllll}
\hline 0.9 & 1 & 1 & 1 & \\
0.8 & 0.7 & 0.5 & 0.3
\end{tabular}

\section{Figure 4}

Model gene expression and nomogram. (A, B) Verification of 2 model gene expression in CRC and normal tissue using the HPA database; (C) Nomogram of predicting 1-, 2-, and 3-years OS of CRC patients in the TCGA database. 
A

TCGA

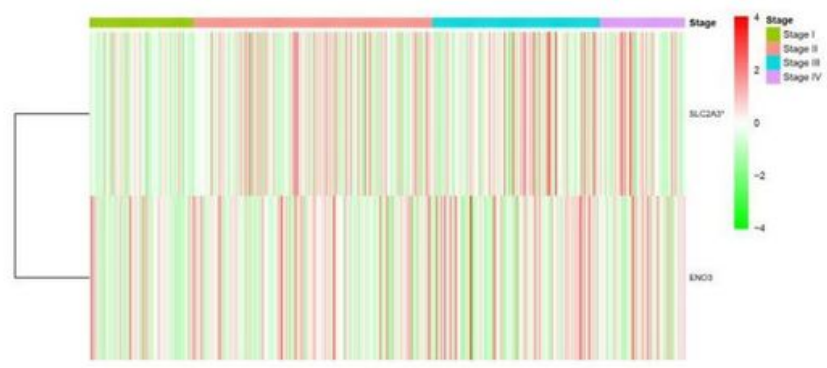

C

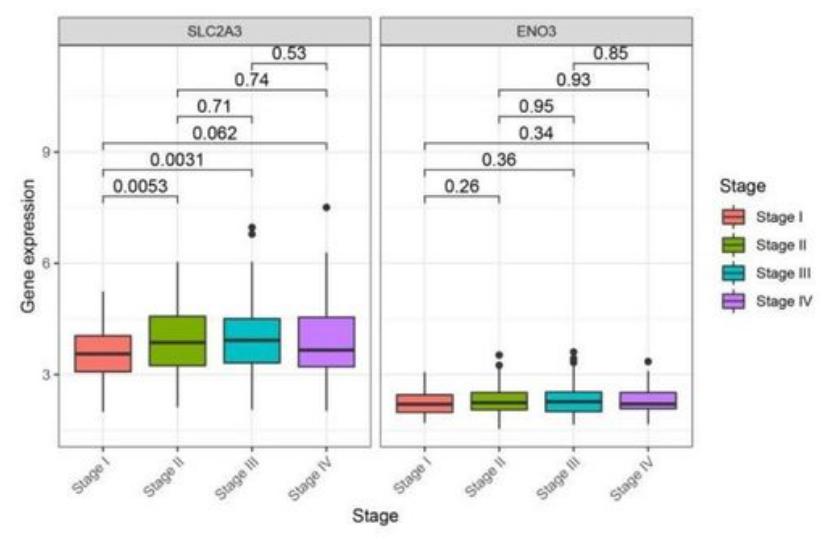

B

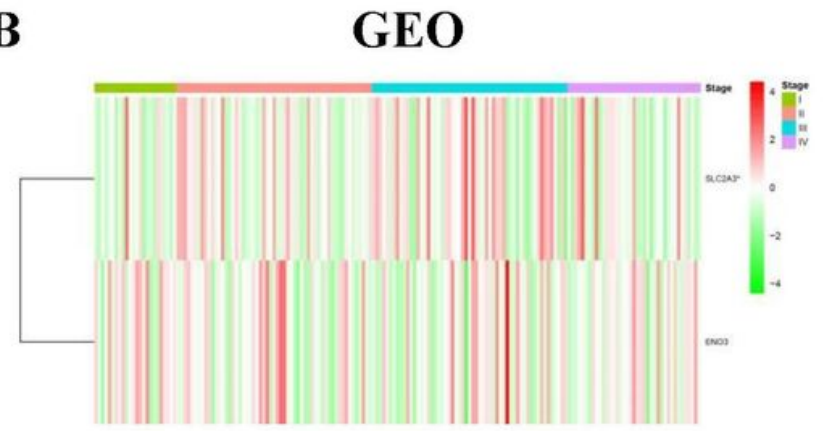

D

GEO

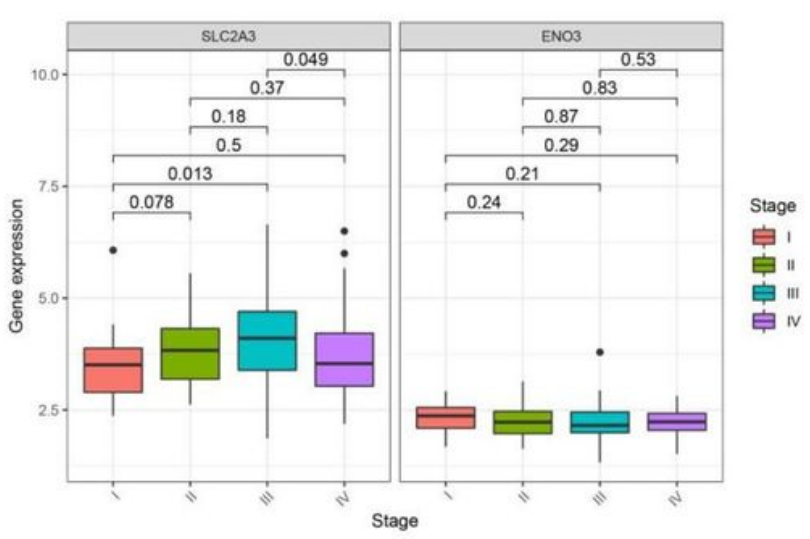

Figure 5

Clinical correlation of model gene expression. (A, B) Heatmaps of the gene expression profile of 2 model gene in different stages from the TCGA and GEO database; (C, D) The expression level of 2 model gene with different stages. 
A
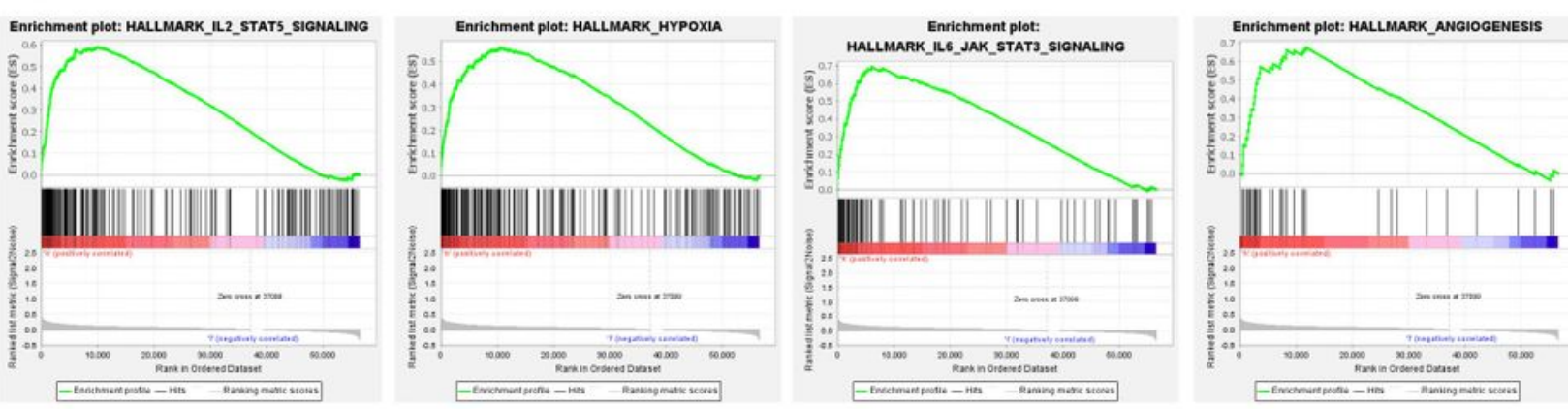

B
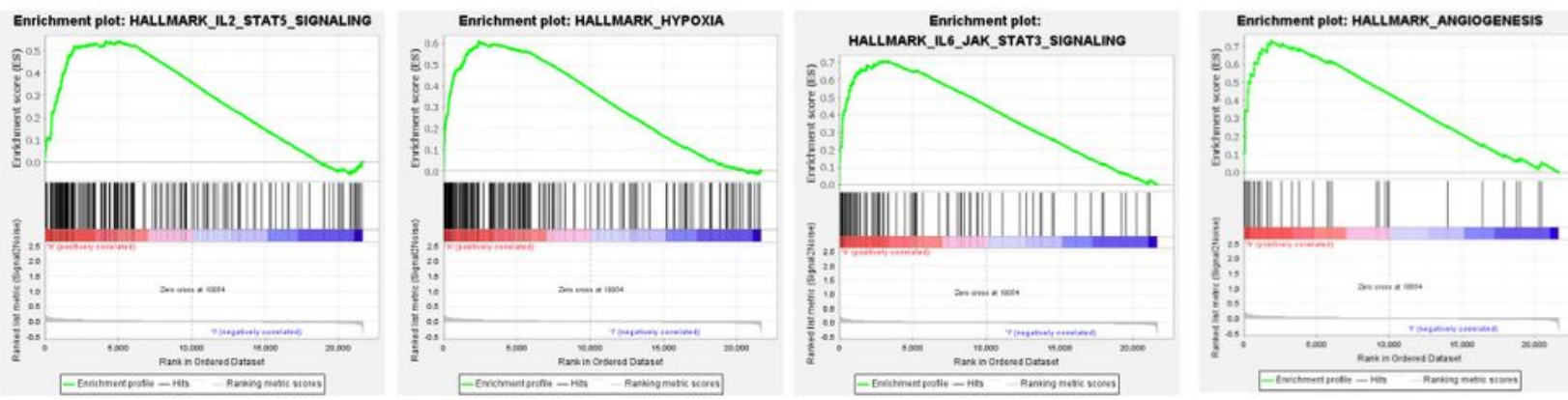

C
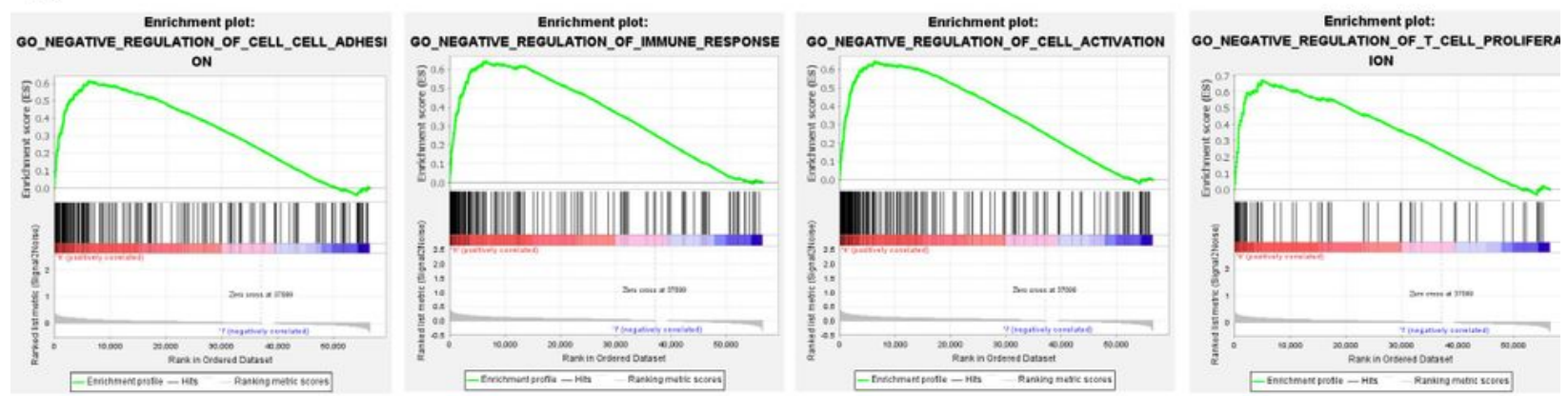

D
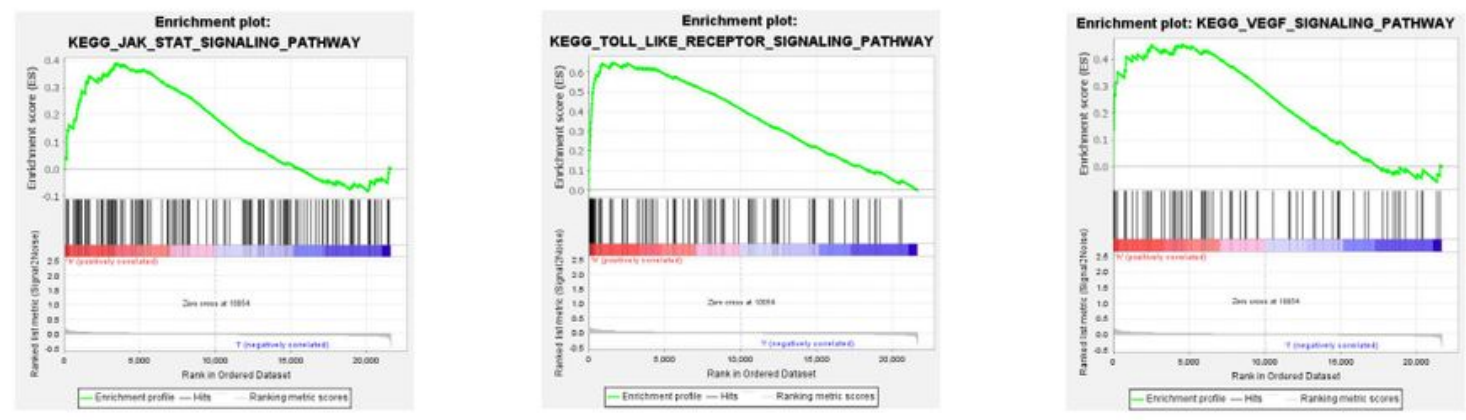

Figure 6

GSEA enrichment between low and high hypoxia risk groups. (A, B) Hypoxia-related signaling enrichment analysis of TCGA and GEO database; (C) GO enrichment analysis and (D) KEGG pathway of TCGA database. 
A

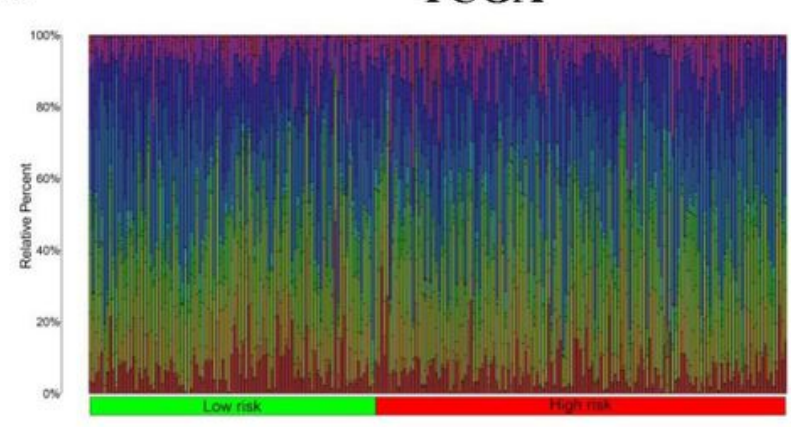

GEO

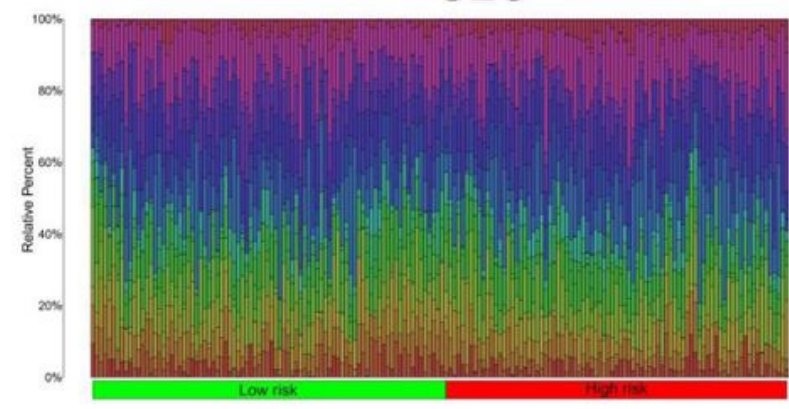

C

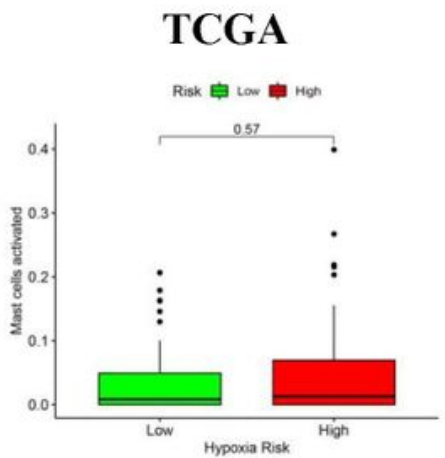

GEO

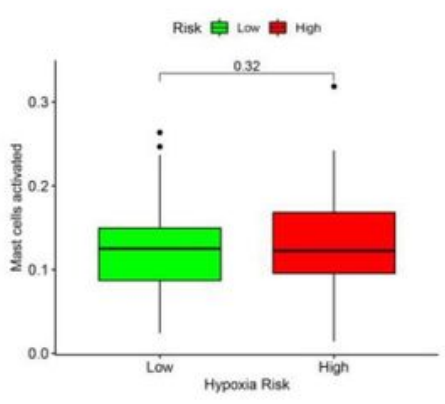

D

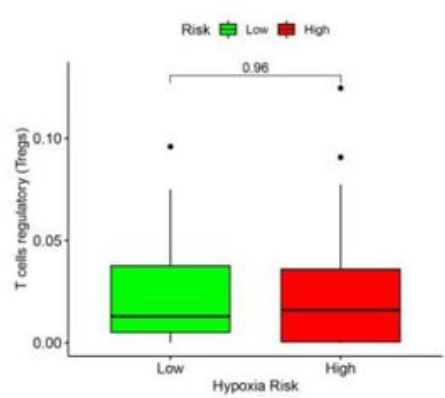

GEO

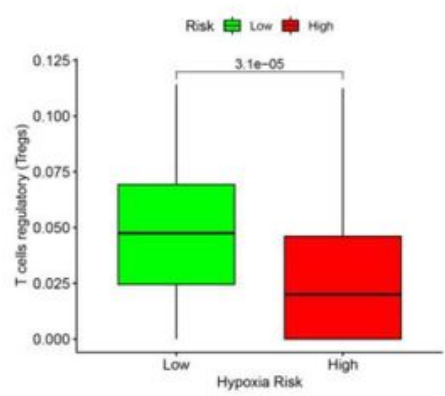

B
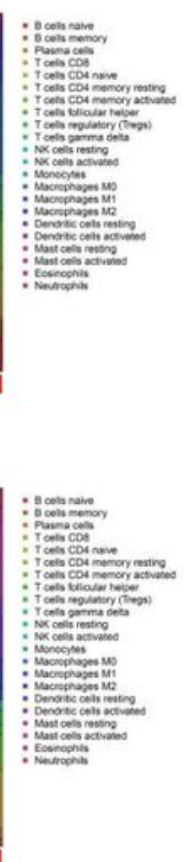

E

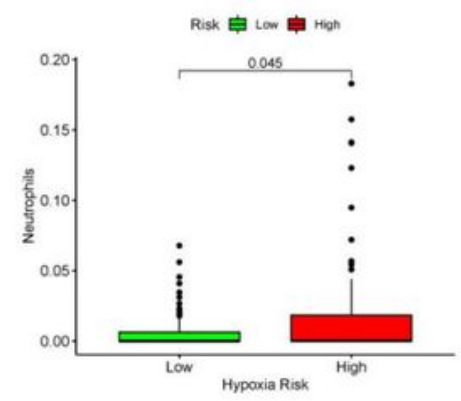

\section{GEO}

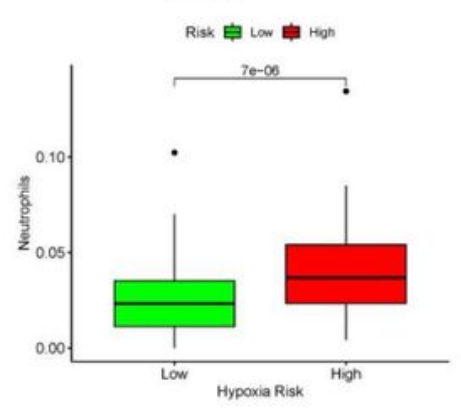

Figure 7

Immune landscape between low and high hypoxia risk groups. (A) Relative proportion of immune cells and (B-E) significant difference of immune cell infiltration in patients with low and high risk of hypoxia 
A

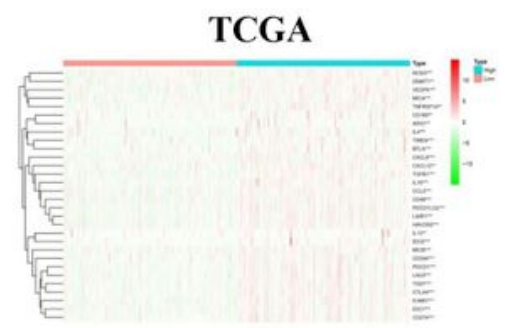

C
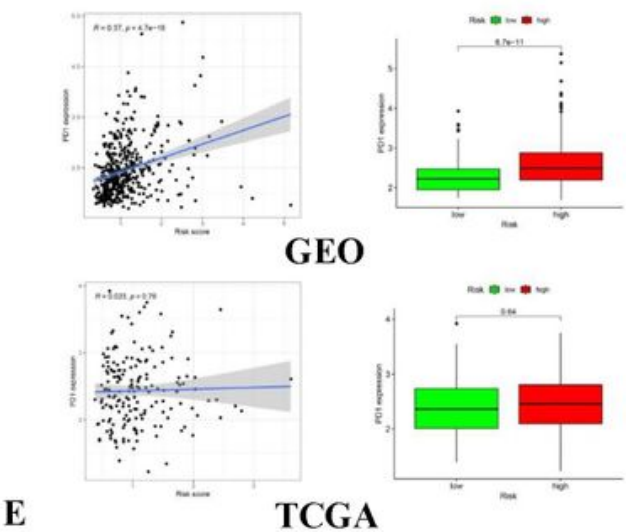

E

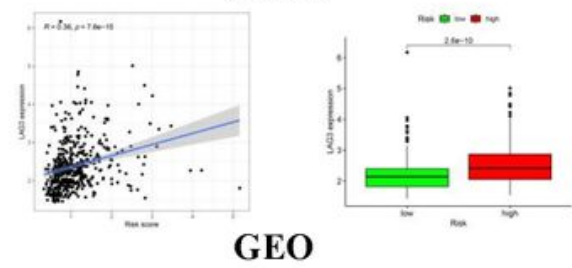

G
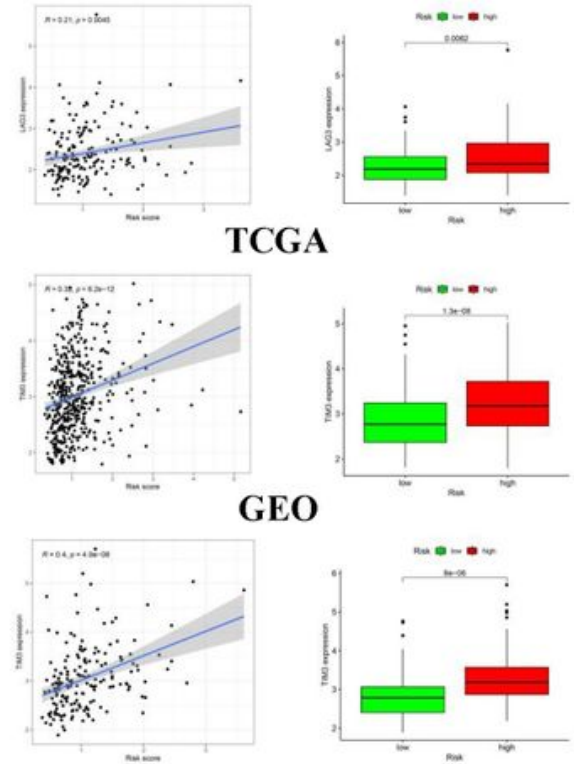

B

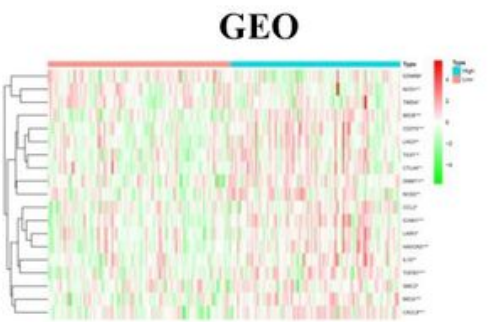

D

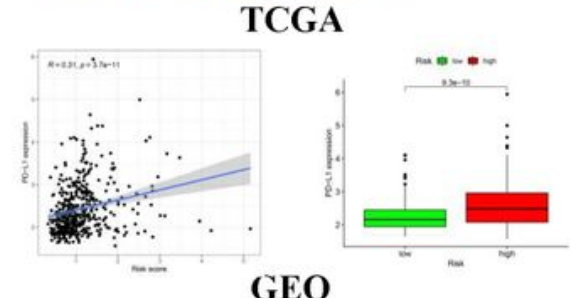

$\mathbf{F}$

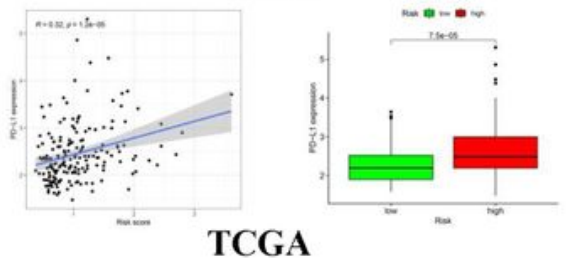

TCGA

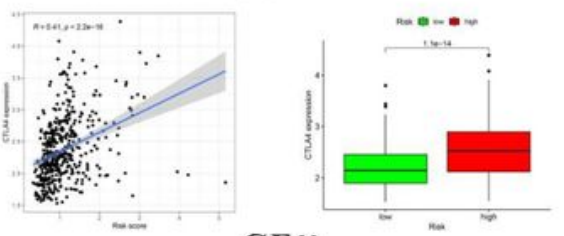

GEO
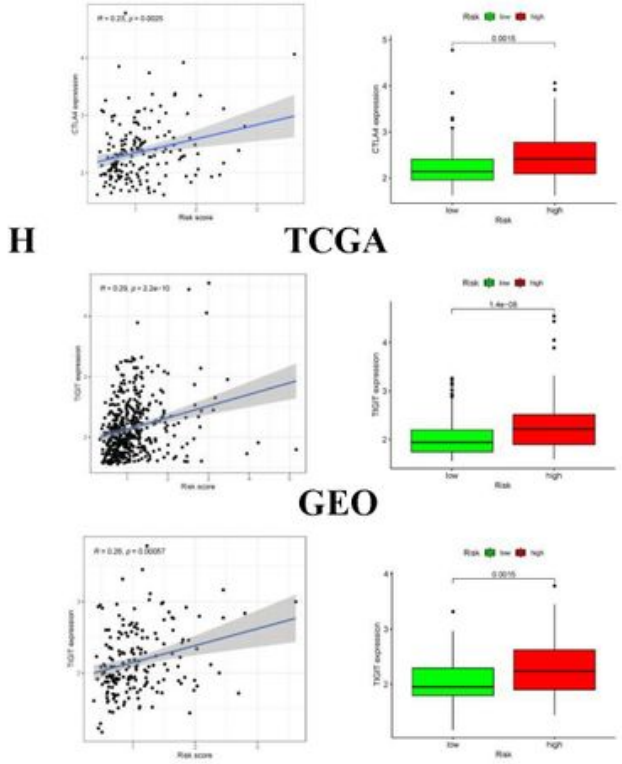

\section{Figure 8}

Expression of immunosuppressive genes in low and high-risk hypoxia groups. (A, B) The gene profiles involved in the negative regulation of the Cancer-Immunity Cycle and $(\mathrm{C}-\mathrm{H})$ immune checkpoints expression level in low and high hypoxia risk groups in the TCGA and GEO databases. 